\title{
Produção científica sobre Recursos Educacionais Abertos ${ }^{1}$
}

\author{
Scientific production about Open \\ Educational Resources
}

Jimena de Mello HEREDIA²

Rosângela Schwarz RODRIGUES 3

Eleonora Milano Falcão VIEIRA4

\section{Resumo}

Identifica os artigos publicados em periódicos indexados na Web of Science, para caracterizar a produção científica sobre Recursos Educacionais Abertos, no âmbito do Ensino Superior. A metodologia é exploratória, descritiva e de abordagem quanti-qualitativa, constituindo o corpus por meio da estratégia de levantamento, cujos dados foram analisados descritivamente e por meio da técnica de análise de conteúdo. Identificaram-se 115 artigos de autoria de 243 pesquisadores, publicados em 43 periódicos, entre 2008 e 2014. Verificou-se que $67 \%$ dos periódicos nos quais se publicou o assunto são de acesso pago, concentrando 56\% dos artigos em acesso restrito. As instituições do Reino Unido, Espanha e Canadá que mais concentram pesquisadores que publicaram sobre Recursos Educacionais Abertos são todas especializadas em Educação a Distância. Predominaram os autores que atuam na área da Educação (48\%), da Computação (22\%) e das Engenharias (11\%). Na etapa qualitativa, descartaram-se 6 artigos por não constituírem iniciativas de Ensino Superior, de modo que a análise de conteúdo se centrou em 99 artigos em inglês, 8 em espanhol e 2 em português, totalizando 109 artigos analisados na íntegra. Os artigos foram classificados em 7 categorias: 21\% recuperação e repositórios; 19\% desafios; 16\% tecnologias; 14\% produção; 13\% políticas de incentivo e sustentabilidade; 10\% adaptação e reúso; e 4\% open courseware. O núcleo das publicações concentra-se em um periódico canadense (28\% dos artigos) e em 26 periódicos (60\%) da área da educação.

Palavras-chave: Acesso aberto. Produção científica. Recursos educacionais abertos.

\begin{abstract}
The present research identifies articles published in journals indexed in the Web of Science to characterize the scientific production on Open Educational Resources, in the higher education area. Descriptive and exploratory methodology of a quantitative and qualitative approach used mixed methods, constituting the research corpus by a survey strategy whose data was analyzed descriptively and through content analysis technique. As a result, it was possible to identify 115 articles of 243 researchers, published in 43 journals between 2008 and 2014. It was found that $67 \%$ of the journals with Open Educational Resources publications are of paid access, concentrating 56\% of the articles in a restricted access. Institutions in the United Kingdom, Spain and Canada with researchers who have published on Open Educational Resources are all specialized in Distance Education. There was a predominance of authors working in the area of Education

\footnotetext{
1 Artigo elaborado a partir da dissertação de J.M. HEREDIA, intitulada"Recursos Educacionais Abertos:mapeamento da comunicação científica". Universidade Federal de Santa Catarina, 2015.

2 Universidade Federal de Santa Catarina, Centro Socioeconômico, Laboratório de Mídias Integradas, Campus Reitor João David Ferreira Lima, Centro Socioeconômico, Bloco F., 88040-900, Florianópolis, SC, Brasil. Correspondência para/Correspondence to: J.M. HEREDIA. E-mail:<jimenamello@gmail.com>.

3 Universidade Federal de Santa Catarina, Departamento de Ciência da Informação, Programa de Pós-Graduação em Ciência da Informação. Florianópolis, SC, Brasil.

${ }^{4}$ Universidade Federal de Santa Catarina, Laboratório de Mídias Integradas, Departamento de Ciências Contábeis. Florianópolis, SC, Brasil.

Recebido em 25/11/2015, reapresentado em 4/5/2016 e aprovado para publicação em 30/6/2016.
} 
(48\%), Computing (22\%) and Engineering (11\%) in comparison to other areas. In the qualitative stage, six articles were discarded so that the content analysis focused on 99 articles in English, eight in Spanish and two in Portuguese, totaling 109 articles analyzed in full. The articles were divided into seven categories: $21 \%$ of recovery and repositories, $19 \%$ of challenges, $16 \%$ of technologies, $14 \%$ of production, $13 \%$ on incentive policies and sustainability, $10 \%$ of adaptation and reuse and $4 \%$ on open courseware. It is possible to conclude that publications core focuses on a Canadian journal and 26 journals about education.

Keywords: Open access. Scientific production. Open educational resources.

\section{Introdução}

Como veículos formais de disseminação científica, os periódicos são instrumentos essenciais para identificar e mensurar a produção científica de um país ou a área de conhecimento, capazes de orientar políticas, programas e sistemas de fomento à pesquisa científica (PACKER, 2011; WHITLEY, 2000). Por meio da análise de artigos publicados em títulos reconhecidos, pode-se analisar a situação das pesquisas em determinada área do conhecimento ou tema. Reconhece-se, entretanto, que os periódicos científicos não são o único meio para a divulgação científica, pois cada área define formas próprias de pesquisa e níveis de cooperação, delineando o que é tido como aceitável em seu âmbito (MEADOWS, 1999; MUELLER, 2007).

O acesso aberto (open access) às publicações científicas é a parte que reivindica a "construção de um domínio público para a ciência e a cultura, que permita a difusão e a reutilização do conhecimento e, por extensão, um rápido progresso científico e cultural" (ABADAL, 2012, p.8). O movimento de Acesso Aberto à Ciência foi influenciado por condições econômicas. Na década de 1990, o aumento dos preços das revistas superava a inflação. Mueller (2007) conta que, durante as décadas de 1970 e 1980, as editoras comerciais aumentaram despropositadamente o valor de suas assinaturas, resultando na insustentabilidade de sua manutenção pelas bibliotecas. Essa "crise dos periódicos", como ficou conhecida, significou, e certamente ainda significa, um "apartheid cognitivo", visto que os países em desenvolvimento acabam ficando mais distantes da informação científica e médica atualizada - cerca de 70\% da população mundial é afetada de alguma forma (GUEDÓN, 2001).

Em relação à educação e ao ensino, iniciativas afins ao movimento do acesso aberto incluem a publicação de materiais e conteúdos docentes, permitindo a construção de um modelo pedagógico que busca promover o uso, o reúso e o compartilhamento desses recursos por estudantes, docentes e comunidade em geral (SANTOS-HERMOSA et al., 2012). Esses materiais se denominam Open Educational Resources (OER, Recursos Educacionais Abertos), os quais, por meio das tecnologias digitais de informação e comunicação, são disponibilizados para consulta, utilização e adaptação por uma comunidade de usuários para fins não comerciais (BUTCHER, 2011). Pode-se afirmar que o movimento OER obteve êxito ao estimular e renovar o interesse acerca da abertura em educação, configurando o "estopim para inúmeros movimentos que hoje usam o termo 'aberto'” (AMIEL; SOARES, 2015, p. 116).

Nesse panorama, este trabalho coloca a questão: quais as características das produções científicas sobre OER no âmbito do Ensino Superior? Tem-se como objetivo geral, portanto, caracterizar a produção científica sobre OER no âmbito do Ensino Superior, considerando os artigos publicados em periódicos indexados em todas as bases da plataforma Web of Science (WoS). Para tanto, determinaram-se os seguintes objetivos específicos: a) identificar os artigos que abordaram o tema OER referentes ao ensino superior, a partir de todas as bases da plataforma WoS; b) descrever os periódicos que publicaram artigos sobre OER; c) identificar os países, as instituições e a área de atuação dos autores que publicaram sobre OER no período pesquisado; e d) analisar o conteúdo dos artigos publicados.

\section{Revisão de literatura}

Duas iniciativas importantes sinalizaram o início do movimento OER, em 2001 (GUTIÉRREZ et al., 2014). A primeira consistiu na criação do Creative Commons, ferramenta de licenças que possibilita ao detentor dos direitos autorais estabelecer de quais deles abdicará, possibilitando cópia, tradução, adaptação e compartilhamento livre do seu material (D'ANTONI, 2009; SANTOS, 
2013). A segunda foi a iniciativa do Massachusets Institute of Technology, também em 2001, de disponibilizar seus recursos digitais para o ensino, aprendizagem e pesquisa para seus estudantes e para o mundo inteiro por meio da Internet, com a criação do Open Course Ware (OCW) (ABELSON, 2008; LANE, 2009; SANTOS-HERMOSA et al., 2012; TOVAR; PIEDRA, 2014; GUTIÉRREZ et al., 2014).

O conceito de OER é abrangente e não definido universalmente. Faltam fundamentos teóricos e filosóficos que sustentem essa forma de produção social de conhecimento (DEIMANN; FARROW, 2013). Costa (2012) afirma que uma multiplicidade de objetos e de materiais digitais on-line acaba sendo classificada como OER, visto que sua definição tem se modificado à medida que a tecnologia evolui e surgem novos usos.

As principais tensões existentes em relação ao conceito de OER (CAMILLERI et al., 2014) são: (a) natureza do recurso - muitas definições limitam o conceito de OER a recursos digitais, porém, outras consideram que qualquer recurso pode ser incluído na definição; (b) fonte do recurso - ao passo que algumas definições requerem que o OER seja produzido com o objetivo educacional explícito, outras incluem aqueles que têm apenas potencial para a aprendizagem; (c) nível de abertura - a maioria das definições implica o recurso estar em domínio público, enquanto outras requerem o uso para fins educacionais ou excluem fins comerciais.

Desde a implementação da primeira iniciativa, as preocupações acerca dos OER se modificaram (SANTOS-HERMOSA et al., 2012). No princípio, a meta principal era garantir e/ou facilitar o acesso aos materiais criados, especialmente por meio de repositórios - chamados repositórios educacionais. Sendo o meio de acesso aos OER, os repositórios permitem a preservação, reutilização e acesso permanente, assim como maior visibilidade e facilidade de busca e recuperação. É principalmente por meio dos repositórios que os usuários podem interagir com os OER - acessando-os, reutilizando-os e até os recriando. Uma das oportunidades que um repositório permite é dar acesso a materiais da melhor qualidade (ROLFE, 2012).

Essas ações fazem despontar uma segunda fase do desenvolvimento dos OER, cuja ênfase está no seu uso e integração às práticas docentes. É por isso que se considera que os repositórios estão no centro de seu crescimento (SANTOS-HERMOSA et al., 2012; ROLFE, 2012).

Ao valorizar práticas de aprendizagem mais próximas à cultura da web e da sociedade do conhecimento, os OER estimulam e fortalecem a autoria, pois escolher como compartilhar um recurso criado é uma decisão que prescinde de mediadores como, por exemplo, as editoras (ROSSINI; GONZALEZ, 2012).

A maior utilização das tecnologias móveis pode ser vista como uma maneira de fomentar o uso dos OER, uma vez que facilitam questões relacionadas à conectividade da Internet (MULDER, 2008).

\section{Procedimentos metodológicos}

Esta pesquisa é exploratória, descritiva e de abordagem quanti-qualitativa, utilizando de métodos mistos. Seu corpus foi constituído por meio da estratégia de levantamento, sendo os dados analisados descritivamente e por meio da técnica de análise de conteúdo (BRAGA, 2007; CERVO et al., 2007; CRESWEL, 2010). O corpus da pesquisa é constituído pelos artigos recuperados a partir de todas as bases da plataforma WoS.

Para realizar a etapa quantitativa da pesquisa, correspondente aos objetivos específicos (a), (b) e (c), utilizou-se, como instrumento um formulário Excel para a coleta de dados de 14 itens para cada artigo recuperado. As informações referentes aos periódicos foram coletadas na edição 2013 do Journal Citacion Reports (JCR) e nas páginas dos próprios periódicos. Já para alcançar o objetivo (d), referente à etapa qualitativa do trabalho, utilizou-se um formulário Excel, com vistas a constituir fichas documentais, fundamentais à análise de conteúdo.

Considerando o viés qualitativo de parte desta pesquisa e os procedimentos empregados, interpretam-se os resultados da análise dos textos no intuito de extrair o seu significado (CRESWEL, 2010). Braga (2007) aponta que a análise de conteúdo é indicada em pesquisas exploratórias. Ela é compreendida como um método qualitativo de pesquisa, pelo qual se efetua uma análise sistemática da ocorrência de palavras, frases e conceitos em materiais diversos (CONONNAY; POWELL, 2010). 
Conforme Bardin (2011), os dados brutos do texto são recortados, agregados e enumerados segundo uma unidade que pode representar o respectivo conteúdo. Considerando a estrutura dos artigos do corpus e os objetivos específicos, estabeleceram-se as seguintes unidades de registro para esta pesquisa: (a) palavras-chave; (b) objetivo; (c) amostra; (d) metodologia; e (e) resultados.

\section{Resultados e Discussão}

A busca foi finalizada em fevereiro de 2015 na WoS, visando contemplar os artigos publicados em fascículos referentes ao ano de 2014. Essa consulta, usando a estratégia "Open Educational Resources" em tópico OR "OER" em título, resultou em 115 artigos, os quais constituíram o corpus da pesquisa na etapa quantitativa.

Como não foram efetuados recortes temporais na estratégia de busca, foi possível constatar quão recentes são os artigos analisados nesta amostra: as primeiras publicações, correspondentes a 3 artigos, datam do ano de 2008; 8 artigos foram identificados em 2009; 9 em 2010; 12 em 2011; 27 em 2012; 33 em 2013; e 23 em 2014. Os dados identificados reiteram a tendência de crescimento do tema a partir de 2008.

Além do que foi levantado por meio da amostra, destaca-se que, embora o termo OER tenha sido cunhado em 2002, no Forum on the Impact of Open Courseware for Higher Education in Developing Countries, da United Nations Educational Scientific and Cultural Organization, (2002) (Unesco, Organização das Nações Unidas para a Educação, a Ciência e a Cultura) (D'ANTONI, 2009), o primeiro artigo com a expressão OER foi publicado em 2005: "OpenSeminar: A web-based collaboration tool for open educational resources", na International Conference on Collaborative Computing.

\section{Periódicos que publicaram artigos sobre OER}

Os 115 artigos que compõem o corpus foram publicados em 43 periódicos científicos diferentes. No Quadro 1 apresentam-se os títulos em ordem decrescente, segundo o número de artigos publicados, descrevendo o país do periódico, a entidade editora, a área de conhecimento, o Índice-H e o Fator de Impacto.
Verificou-se que os periódicos analisados provêm de 16 países, sendo Reino Unido e Estados Unidos aqueles com maior número de artigos sobre OER, com 14 e 10 periódicos, respectivamente. Os 24 periódicos de ambos os países compõem mais da metade $(55,8 \%)$ daqueles que integram esta pesquisa e contabilizam 60 artigos. Possuem Índice-H médio de 31 e Fator de Impacto médio de 2.051. O acesso a tais periódicos é pago por subscrição com opção de Acesso Aberto (10 ingleses e 9 estadunidenses), não sendo encontrado nenhum de acesso aberto.

O Brasil aparece em terceiro lugar no que diz respeito ao número de periódicos que publicaram artigos sobre OER. Destaca-se que os três periódicos brasileiros - "Informação \& Sociedade, Educação \& Sociedade" e "Perspectivas em Ciência da Informação" - são todos de acesso aberto, apresentando Índice-H médio de 6 e Fator de Impacto médio de 0.169.

Com relação ao total de artigos publicados por periódico, a configuração dos 16 países se modifica. Nesse caso, Reino Unido, Canadá e Estados Unidos têm mais artigos publicados: 36, 33 e 24, respectivamente. Os 33 artigos do Canadá foram publicados em um só periódico, o International Review of Research in Open and Distributed Learning, o único do país a publicar sobre OER. Isso demonstra a existência de um título de criação recente, em 2000, e em acesso aberto, que concentra as discussões sobre OER, responsável por 28\% do total de artigos da amostra.

Quanto à área de conhecimento, constatou-se que 10 periódicos são classificados em 2, 3 ou 4 áreas de conhecimento da WoS. Para fins de análise, optou-se por agrupar as áreas descritas nos periódicos em grandes grupos, de acordo áreas gerais de conhecimento.

Em se tratando da produtividade de um periódico, é por meio dessa aferição que se estabelecem o núcleo e as áreas de dispersão sobre um determinado assunto em um conjunto de revistas (VANTI, 2010). Dessa forma, confirma-se aqui a Lei de Bradford, verificando-se a predominância dos periódicos da área de Educação, correspondentes a $26(60,0)$ do total dos 43 periódicos, nos quais se publicaram 95 (82,6\%) do total de 115 artigos sobre OER. Os periódicos da área de Educação têm Índice-h entre 2 e 97, sendo o valor médio de 21,6. O seu Fator de Impacto varia de 0.228 a 3.468. Constatou-se, portanto, a discrepância entre os valores, o que é ainda 
Quadro 1. Periódicos em que os artigos do corpus foram publicados.

1 de 2

\begin{tabular}{|c|c|c|c|c|c|c|c|}
\hline Título & $\begin{array}{c}\mathrm{n}^{\circ} \\
\text { artigos }\end{array}$ & País & $\begin{array}{l}\text { Entidade Editora } \\
\text { (Publisher) }\end{array}$ & Área de conhecimento & $\begin{array}{l}\text { Tipo de } \\
\text { acesso }\end{array}$ & Índice-H & $\begin{array}{l}\text { Fator de } \\
\text { impacto }\end{array}$ \\
\hline $\begin{array}{l}\text { International Review of Research } \\
\text { in Open and Distance Learning }\end{array}$ & 33 & $C A$ & Athabasca University Press & $\begin{array}{l}\text { Education \& Educational } \\
\text { Research }\end{array}$ & $\mathrm{OA}$ & 8 & 0.748 \\
\hline Distance Education & 14 & GB & $\begin{array}{l}\text { Routledge Journals, Taylor } \\
\text { \& Francis Ltd. }\end{array}$ & $\begin{array}{l}\text { Education \& Educational } \\
\text { Research }\end{array}$ & so & 10 & 0.725 \\
\hline $\begin{array}{l}\text { IEEE Transactions on Learning } \\
\text { Technologies }\end{array}$ & 7 & US & IEEE Computer Soc. & $\begin{array}{l}\text { Education \& Educational } \\
\text { Research }\end{array}$ & so & 11 & 1.220 \\
\hline IEEE Transactions on Education & 6 & US & $\begin{array}{l}\text { IEEE-Inst. Electrical } \\
\text { Electronics Engineers In }\end{array}$ & $\begin{array}{l}\text { Education, Scientific } \\
\text { Disciplines/Engineering, } \\
\text { Electrical \& Electronic }\end{array}$ & so & 35 & 1.221 \\
\hline $\begin{array}{l}\text { British Journal of Educational } \\
\text { Technology }\end{array}$ & 4 & GB & Wiley-Blackwell & $\begin{array}{l}\text { Education \& Educational } \\
\text { Research }\end{array}$ & SU & 37 & 1.394 \\
\hline $\begin{array}{l}\text { Computer Assisted Language } \\
\text { Learning }\end{array}$ & 4 & GB & $\begin{array}{l}\text { Routledge Journals, Taylor } \\
\text { \& Francis Ltd. }\end{array}$ & $\begin{array}{l}\text { Education \& Educational } \\
\text { Research }\end{array}$ & SU & 11 & 0.880 \\
\hline $\begin{array}{l}\text { Journal of Computing In Higher } \\
\text { Education }\end{array}$ & 3 & US & Springer & $\begin{array}{l}\text { Education \& Educational } \\
\text { Research }\end{array}$ & so & 5 & 0.826 \\
\hline Profesional de la Informacion & 3 & ES & $\begin{array}{l}\text { EPI - El Profesional de la } \\
\text { Informacion }\end{array}$ & $\begin{array}{l}\text { Information Science \& } \\
\text { Library Science }\end{array}$ & so & 9 & 0.402 \\
\hline $\begin{array}{l}\text { Australasian Journal of } \\
\text { Educational Technology }\end{array}$ & 2 & $\mathrm{AU}$ & Charles Sturt Univ. & $\begin{array}{l}\text { Education \& Educational } \\
\text { Research }\end{array}$ & $\mathrm{OA}$ & 18 & 0.875 \\
\hline Computers \& Education & 2 & GB & $\begin{array}{l}\text { Pergamon-Elsevier Science } \\
\text { Ltd. }\end{array}$ & $\begin{array}{l}\text { Education \& Educational } \\
\text { Research }\end{array}$ & SO & 61 & 2.630 \\
\hline $\begin{array}{l}\text { Journal of Computer Assisted } \\
\text { Learning }\end{array}$ & 2 & GB & Wiley-Blackwell & $\begin{array}{l}\text { Education \& Educational } \\
\text { Research }\end{array}$ & SO & 41 & 1.023 \\
\hline $\begin{array}{l}\text { Journal of Universal Computer } \\
\text { Science }\end{array}$ & 2 & AT & $\begin{array}{l}\text { Graz Univ. Technolgoy, Inst. } \\
\text { Information Systems } \\
\text { Computer Med. }\end{array}$ & $\begin{array}{l}\text { Computer Science/Software } \\
\text { Engineering Computer } \\
\text { Science/Theory \& Methods }\end{array}$ & OA & 25 & 0.401 \\
\hline Learning Media and Technology & 2 & GB & $\begin{array}{l}\text { Routledge Journals, Taylor \& } \\
\text { Francis Ltd. }\end{array}$ & $\begin{array}{l}\text { Education \& Educational } \\
\text { Research }\end{array}$ & so & 13 & 0.958 \\
\hline Teaching in Higher Education & 2 & US & $\begin{array}{l}\text { Routledge Journals, Taylor \& } \\
\text { Francis Ltd. }\end{array}$ & $\begin{array}{l}\text { Education \& Educational } \\
\text { Research }\end{array}$ & SO & 19 & 0.623 \\
\hline Educational Technology \& Society & 1 & NZ & $\begin{array}{l}\text { IEEE Computer Soc., } \\
\text { Learning Technology Task } \\
\text { Force, Massey University }\end{array}$ & $\begin{array}{l}\text { Education \& Educational } \\
\text { Research }\end{array}$ & OA & 30 & 0.824 \\
\hline Academic Medicine & 1 & US & Lippincott Williams\& Wilkins & $\begin{array}{l}\text { Education/Scientific } \\
\text { Disciplines/Health Care } \\
\text { Sciences \& Services }\end{array}$ & SU & 97 & 3.468 \\
\hline Advanced Science Letters & 1 & $\mathrm{Nl}^{* *}$ & $\begin{array}{l}\text { American Scientific } \\
\text { Publishers }\end{array}$ & Multidisciplinary Sciences & SU & 17 & $\mathrm{Nl}^{* *}$ \\
\hline $\begin{array}{l}\text { Arbor-Ciencia Pensamiento y } \\
\text { Cultura }\end{array}$ & 1 & ES & $\begin{array}{l}\text { Consejo Superior de } \\
\text { Investigaciones Cientificas }\end{array}$ & Arts \& Humanities & OA & 4 & 0.105 \\
\hline Computers in Human Behavior & 1 & US & $\begin{array}{l}\text { Pergamon-Elsevier Science } \\
\text { Ltd. }\end{array}$ & Psychology & SO & 67 & 2.273 \\
\hline Educação \& Sociedade & 1 & BR & $\begin{array}{l}\text { Centro de Estudos de } \\
\text { Educação e Sociedade } \\
\text { Unicamp }\end{array}$ & Edpucation & OA & 12 & 0.3314 \\
\hline $\begin{array}{l}\text { Etr\&D-Educational Technology } \\
\text { Research and Development }\end{array}$ & 1 & US & Springer & $\begin{array}{l}\text { Education \& Educational } \\
\text { Research }\end{array}$ & SO & 49 & 0.919 \\
\hline European Journal of Education & 1 & GB & Wiley-Blackwell & $\begin{array}{l}\text { Education \& Educational } \\
\text { Research }\end{array}$ & SO & 8 & 0.329 \\
\hline
\end{tabular}


Quadro 1. Periódicos em que os artigos do corpus foram publicados.

\begin{tabular}{|c|c|c|c|c|c|c|c|}
\hline Título & $\begin{array}{c}\mathrm{n}^{\circ} \\
\text { artigos }\end{array}$ & País & $\begin{array}{l}\text { Entidade Editora } \\
\text { (Publisher) }\end{array}$ & Área de conhecimento & $\begin{array}{l}\text { Tipo de } \\
\text { acesso }\end{array}$ & Índice-H & $\begin{array}{l}\text { Fator de } \\
\text { impacto }\end{array}$ \\
\hline Formación Universitária & 1 & $\mathrm{CL}$ & $\begin{array}{l}\text { Centro de Informacion } \\
\text { Tecnologica }\end{array}$ & Education Engineering & $\mathrm{OA}$ & 4 & 0.4000 \\
\hline Informação \& Sociedade-Estudos & 1 & BR & $\begin{array}{l}\text { Univ. Federal da Paraíba } \\
\text { CCSA }\end{array}$ & $\begin{array}{l}\text { Information Science \& } \\
\text { Library Science/Sociology/ } \\
\text { Communications }\end{array}$ & $\mathrm{OA}$ & 3 & 0.080 \\
\hline Innovar & 1 & $\mathrm{CO}$ & $\begin{array}{l}\text { Univ Nacional Colombia, Fac } \\
\text { Ciencias Econ }\end{array}$ & $\begin{array}{l}\text { Business Management/ } \\
\text { Public Administration }\end{array}$ & $\mathrm{OA}$ & 4 & 0.0690 \\
\hline $\begin{array}{l}\text { International Journal of } \\
\text { Educational Development }\end{array}$ & 1 & $G B$ & $\begin{array}{l}\text { Pergamon-Elsevier Science } \\
\text { Ltd. }\end{array}$ & $\begin{array}{l}\text { Education \& Educational } \\
\text { Research }\end{array}$ & SU & 24 & 0.841 \\
\hline $\begin{array}{l}\text { International Journal of } \\
\text { Engineering Education }\end{array}$ & 1 & IE & Tempus Publications & $\begin{array}{l}\text { Education Scientific } \\
\text { Disciplines, Engineering, } \\
\text { Multidisciplinary }\end{array}$ & SU & 20 & 0.360 \\
\hline Investigacion Bibliotecologica & 1 & $M X$ & $\begin{array}{l}\text { Univ Nacional Autonoma } \\
\text { Mexico }\end{array}$ & $\begin{array}{l}\text { Information Science \& } \\
\text { Library Science }\end{array}$ & $\mathrm{OA}$ & 3 & 0.062 \\
\hline $\begin{array}{l}\text { Journal of Agricultural \& } \\
\text { Environmental Ethics }\end{array}$ & 1 & $\mathrm{NL}$ & Springer & Ethics & so & 27 & 1.250 \\
\hline $\begin{array}{l}\text { Journal of Science Education and } \\
\text { Technology }\end{array}$ & 1 & US & Springer & $\begin{array}{l}\text { Education \& Educational } \\
\text { Research }\end{array}$ & SO & 14 & 0.869 \\
\hline Meat Science & 1 & GB & Elsevier Sci. Ltd. & Food Science \& Technology & SO & 108 & 2.231 \\
\hline Modern Language Journal & 1 & US & Wiley-Blackwell & Linguistics & so & $54^{*}$ & 1.181 \\
\hline New Educational Review & 1 & $P L$ & $\begin{array}{l}\text { Wydawnictwo Adam } \\
\text { Marszalek }\end{array}$ & $\begin{array}{l}\text { Education \& Educational } \\
\text { Research }\end{array}$ & OA & 4 & $\mathrm{NI}$ \\
\hline Perfiles Educativos & 1 & $M X$ & $\begin{array}{l}\text { Universidad Nacional } \\
\text { Autonoma de Mexico - } \\
\text { Instituto de Investigaciones } \\
\text { sobre la Universidad y la } \\
\text { Educacion }\end{array}$ & Education & OA & 2 & 0.2286 \\
\hline $\begin{array}{l}\text { Perspectivas em Ciência da } \\
\text { Informação }\end{array}$ & 1 & BR & $\begin{array}{l}\text { Escola Ciência da } \\
\text { Informação UFMG }\end{array}$ & $\begin{array}{l}\text { Information Science \& } \\
\text { Library Science }\end{array}$ & $\mathrm{OA}$ & 5 & 0.0970 \\
\hline Perspectives in Public Health & 1 & GB & Sage Publications Ltd. & $\begin{array}{l}\text { Public, Environmental \& } \\
\text { Occupational Health }\end{array}$ & so & 8 & 1.035 \\
\hline Proceedings of The Teee & 1 & US & $\begin{array}{l}\text { IEEE-Inst Electrical } \\
\text { Electronics Engineers Inc. }\end{array}$ & $\begin{array}{l}\text { Engineering, Electrical \& } \\
\text { Electronic }\end{array}$ & SO & $190^{*}$ & 5.466 \\
\hline $\begin{array}{l}\text { Program-Electronic Library and } \\
\text { Information Systems }\end{array}$ & 1 & GB & $\begin{array}{l}\text { Emerald Group Publishing } \\
\text { Limited }\end{array}$ & $\begin{array}{l}\text { Information Science \& } \\
\text { Library Science }\end{array}$ & SU & 11 & 0.091 \\
\hline Public Health & 1 & GB & W.B. Saunders Co. Ltd. & $\begin{array}{l}\text { Public, Environmental \& } \\
\text { Occupational Health }\end{array}$ & so & 47 & 1.475 \\
\hline $\begin{array}{l}\text { Revue Scientifique Et Technique- } \\
\text { Office International Des } \\
\text { Epizooties }\end{array}$ & 1 & $F R$ & Office Int. Epizooties & Veterinary Sciences & SU & 35 & $\mathrm{NI}^{* *}$ \\
\hline System & 1 & GB & Elsevier Sci. Ltd. & $\begin{array}{l}\text { Education \& Educational } \\
\text { Research/Linguistics }\end{array}$ & SO & 14 & 0.889 \\
\hline $\begin{array}{l}\text { Technology Pedagogy and } \\
\text { Education }\end{array}$ & 1 & GB & $\begin{array}{l}\text { Routledge Journals, Taylor \& } \\
\text { Francis Ltd. }\end{array}$ & $\begin{array}{l}\text { Education - Teaching } \\
\text { Methods And Curriculum }\end{array}$ & SO & 7 & 0.541 \\
\hline $\begin{array}{l}\text { Turkish Online Journal of } \\
\text { Educational Technology }\end{array}$ & 1 & TR & Sakarya Universitesi & Education Tecnology & $\mathrm{OA}$ & 10 & $\mathrm{NI}^{* *}$ \\
\hline
\end{tabular}

Notas: "Resultado obtido a partir do refinamento por tipo de documento (artigos). ${ }^{* *}$ Dados não identificados nas fontes consultadas. ${ }^{* * *}$ Descrevem-se os países por meio de siglas que observam a ISO 3166-2: AT: Austria; AU: Austrália; BR: Brasil; CA: Canadá; CL: Chile; CO: Colômbia; ES: Espanha; FR: França; GB: Reino Unido; IE: Irlanda; MX: México; NL: Holanda; NZ: Nova Zelândia; PL: Polônia; TR: Turquia; US: Estados Unidos. OA: Open Access; SU: Subscription (para subscrição paga); SO: Subscriçăo paga com opção de Acesso Aberto. Fonte: Web of Science v.5.14, Journal Citation Reports 4.5, SciELO (Scientific Eletronic Library Online) páginas dos periódicos (2014). 
mais evidente quando analisados segundo o tipo de acesso. Percebeu-se a dispersão do tema nos periódicos das demais áreas: Engenharia, Ciência e Tecnologia, Ciência da Informação, Saúde, Humanas, Linguística, Multidisciplinar e outras áreas. Na área de Ciência e Tecnologia, não há nenhum periódico de acesso aberto. Os restritos, no entanto, reúnem os mais altos índices blibliométricos desta pesquisa, contemplando Índice-H entre 17 e 108 e Fator de Impacto de até 3.468. Quanto aos periódicos da área de Ciência da Informação, eles apresentam Índices-H entre 3 e 11 e Fator de Impacto máximo de 0.0970.

Identificou-se que 14 (32,6\%) dos 43 periódicos levantados são de acesso aberto, ao passo que os demais são de acesso restrito: 21 pagos por subscrição com opção de acesso aberto (48,8\%) e 8 (18,6\%) pagos por subscrição. Esses 14 periódicos de acesso aberto provêm de 11 países: Canadá, Austrália, Áustria, Nova Zelândia, Espanha, Brasil, Chile, Colômbia, México, Polônia e Turquia.

Embora o periódico que mais tenha concentrado publicações a respeito seja de acesso aberto, com 33 artigos publicados (28\%), 67 artigos do corpus (58\%) são de acesso por subscrição. Entre os periódicos da área de Educação, constatou-se que 8 são de acesso aberto - correspondendo a 30\% da totalidade das publicações da área. Isoladamente, a sua média de Índice-H é 11 e seu Fator de Impacto mais alto de 0.748. Os periódicos da área de Ciência da Informação de acesso aberto são 3 e apresentam Índice-H entre 3 e 5 e Fator de Impacto de até 0.0970 .

Verificou-se que quase todas (13) as entidades editoriais dos periódicos de acesso aberto são universidades (11) ou centros de pesquisa (2) - exceto a editora polonesa da revistaNewEducational Review, a Wydawnictwo Adam Marszalek. Com relação às entidades editoras comerciais dos periódicos, identificou-se que elas detêm 28 publicações, nos quais foram divulgados 65 artigos. A Tabela 1 detalha esses números por entidade editora.

Quando se observa o Índice-H dos periódicos, é possível verificar que aqueles que publicaram poucos artigos sobre OER possuem os índices mais altos. Os três periódicos cujos Índices-H são os mais altos, o Proceedings of the IEE (Índice-H 190), o Meat Science (Índice-H 108) e o Academic Medicine (Índice-H 97), não são especiali-
Tabela 1. Entidades editoras comerciais.

\begin{tabular}{lcc}
\hline Entidade editora (Publisher) & $\begin{array}{c}\mathrm{n}^{\circ} \\
\text { Periódicos }\end{array}$ & $\begin{array}{c}\mathrm{n}^{\circ} \\
\text { Artigos }\end{array}$ \\
\hline Routledge Journals, Taylor \& Francis Ltd. & 5 & 23 \\
Wiley-Blackwell & 4 & 8 \\
Springer & 4 & 6 \\
IEEE-Inst. Electrical Electronics Engineers In. & 2 & 7 \\
IEEE Computer Soc. & 1 & 7 \\
Pergamon-Elsevier Science Ltd. & 3 & 3 \\
EPI-El Profesional de la Informacion & 1 & 3 \\
Elsevier Sci. Ltd. & 2 & 2 \\
American Scientific Publishers & 1 & 1 \\
Emerald Group Publishing Limited & 1 & 1 \\
Lippincott Williams \& Wilkins & 1 & 1 \\
Office Int Epizooties & 1 & 1 \\
Tempus Publications & 1 & 1 \\
W.B. Saunders Co. Ltd. & 1 & 1 \\
\hline Total & 28 & 65 \\
\hline
\end{tabular}

Fonte: Journal Citacion Reports 4.5 (2014).

zados na área de conhecimento mais proeminente dentre os pesquisados - a da educação -, mas nas áreas de Engenharia, Ciência de Alimentos e Saúde, respectivamente.

A análise efetuada nesta pesquisa visa monitorar o desenvolvimento científico de uma temática - os OER - e identificá-lo no contexto do movimento de acesso aberto. Verificou-se que a maioria (33) dos periódicos provém de países europeus, anglo-saxões e/ou de língua inglesa, os quais publicam em idioma inglês e ratificam a tradição de concentração das publicações em editoras comerciais nos periódicos com maiores indicadores de uso.

Constatou-se que os 43 periódicos, cujos artigos compõem o corpus da pesquisa, são de 16 países, sendo 13 do Reino Unido. O periódico com maior concentração de artigos é canadense, da área de Educação e em acesso aberto. Verificou-se que o núcleo das publicações se concentra na área de Educação (26 periódicos e 95 artigos), dispersando-se gradativamente nas áreas de Engenharia (4 periódicos e 10 artigos), Ciência e Tecnologia (5 periódicos e 10 artigos), Ciência da Informação (5 periódicos e 7 artigos), Saúde (3 periódicos e 3 artigos), Humanas (3 periódicos e 3 artigos), Linguística (2 periódicos e 2 artigos) e Multidisciplinar (2 periódicos e 2 artigos), entre outras (9 periódicos e 11 artigos). Destaca-se que a maior parte (29) dos periódicos nos quais se 
publicou sobre OER é de acesso pago ou por subscrição paga com opção de acesso aberto, concentrando 56\% dos artigos em acesso restrito. Cabe ressalvar que a amostra dessa análise corresponde ao tema da pesquisa, relativamente recente, combinado à estratégia de busca, restrita aos artigos da plataforma WoS.

\section{Autores que publicam sobre OER}

Nesta seção, verificou-se que somam 243 os autores dos 115 artigos que compõem o corpus desta pesquisa. Dentre eles, $88,00 \%$ têm apenas um artigo publicado sobre OER. Identificou-se que 1 autor $(0,41 \%)$ tem 5 artigos publicados, 1 autor $(0,41 \%)$ tem 4 artigos publicados, 1 autor $(0,41 \%)$ tem 3 artigos publicados, 26 autores (10,69\%) têm 2 artigos publicados e 214 autores (88,06\%) têm 1 artigo publicado.

Essas constatações vão ao encontro da Lei de Lotka, que sustenta que poucos autores publicam uma grande proporção da literatura científica e que, quanto mais assim o fizerem, mais terão facilidade para publicar novamente (ARAÚJO, 2006; FERREIRA, 2010). Os três autores da amostra que mais publicaram sobre OER, conforme as estratégias de recuperação estabelecidas, são:

-David Wiley, da Brigham Young University: publicou 5 artigos nos periódicos: Educational Technology Research And Development (1 artigo) e International Review Of Research In Open And Distance Learning (4 artigos);

-John Hilton III, da Brigham Young University: publicou 4 artigos no periódico International Review of Research in Open and Distance Learning; e

-Andy Lane, da Open University: publicou 3 artigos nos periódicos Distance Education (1 artigo), International Review of Research in Open and Distance Learning (1 artigo) e British Journal of Educational Technology (1 artigo).

Atuantes na área de Educação (Wiley e Hilton III) e na área de Ciência da Computação (Lane), os autores mais produtivos publicaram nos periódicos de maior destaque - segundo os critérios desta pesquisa, o International Review of Research In Open and Distance Learning e o Distance Education. Destaca-se que todos os periódicos nos quais esses autores publicaram são da área da Educação, que corresponde ao núcleo das publicações sobre OER.

Dessa forma, constatou-se que 243 autores estão vinculados a 107 instituições de 36 países, e que, das 107 instituições, 96 são universidades.

O Reino Unido tem a maior quantidade de autores que publicaram sobre OER: 54 autores (22\%), de 15 instituições. Nesse caso, e embora se perceba a concentração de autores na Open University (27\%) e na University Southampton (20\%), há mais 13 instituições (86\%) cujos autores publicaram sobre o tema. A terceira autora mais produtiva desta pesquisa é da universidade mais produtiva, a Open University.

Os Estados Unidos têm a segunda maior quantidade de autores que publicaram sobre OER nesta pesquisa: 43 autores (17\%), de 16 instituições. Nesse caso, os autores são distribuídos nas universidades desse país, de onde provêm os mais produtivos desta pesquisa: David Wiley e John Hilton III, da Brigham Young University.

A Espanha tem a terceira maior quantidade de autores que publicaram sobre OER nesta pesquisa: 30 autores (12\%), de 10 instituições. Nesse caso, um terço (10) dos autores se concentra na Universidad Nacional de Educación a Distancia, e todos os demais em universidades.

Embora o periódico mais produtivo desta pesquisa seja o canadense International Review of Research in Open and Distance Learning, o Canadá tem a quarta maior quantidade de autores que publicaram sobre OER: 10 autores (4\%), de 4 instituições. Nesse caso, a maioria (6) dos autores se concentra na Athabasca University, especializada em educação a distância. Um único autor desse país, Paul Stacey, não informou ter vínculo com qualquer instituição.

A Finlândia e o Canadá possuem a quarta maior quantidade de autores que publicaram sobre OER nesta pesquisa: 10 autores (4\%), de 4 instituições, sendo três universidades e uma instituição privada.

Observou-se que as instituições que mais concentram pesquisadores que publicaram sobre OER no Reino Unido, na Espanha e no Canadá são todas especializadas em Educação a Distância: a Open University, a Universidad Nacional de Educación a Distancia e a 
Athabasca University, respectivamente. Esse fato demonstra a importância dos OER nessa modalidade de ensino, dadas as suas potencialidades e o fato de esse tema decorrer de um anterior, do âmbito da educação a distância - os objetos de aprendizagem.

Nesse sentido, pode-se interpretar a distribuição dos autores dos países mais representativos segundo três modelos: a) concentrado em universidades especializadas em Educação a Distância (Reino Unido e Canadá); b) distribuído em universidades de ensino presencial que podem oferecer Educação a Distância simultaneamente (Estados Unidos e Canadá); e c) misto (Espanha), onde ocorrem os dois casos. Nos demais países identificados, destacam-se também as universidades.

Como visto, verificou-se a predominância de instituições que oferecem ensino superior dentre as instituições relacionadas aos autores. Apenas 8 instituições identificadas na amostra desta pesquisa não são universidades. E as universidades que mais se destacam quanto ao número de autores que publicaram sobre OER são: Open University (15 autores), University Southampton (11), Universidad Nacional de Educación a Distancia (10), Rice University (8) Utah State University (7), Universidad de Alicante (6), Athabasca University (6) e Open Universiteit Nederland (6).

Evidencia-se o protagonismo das universidades como produtoras de pesquisa e divulgadoras da produção científica sobre o tema. Esse papel é igualmente de destaque na publicação de periódicos científicos de acesso aberto, pois 11 universidades são entidades editoras dos referidos periódicos de acesso aberto.

Com relação à área de atuação dos autores, utilizaram-se os dados coletados na WoS para levantar tal informação: definiu-se a sua área por meio dos departamentos ou institutos. Embora nessa rubrica constem as informações institucionais dos autores, nem todas são suficientes aos propósitos desta pesquisa: 122 dos 243 autores não dispunham dessa informação naWOS. Assim, procedeu-se à busca da área de atuação desses 122 autores mediante estratégias realizadas por meio do motor de busca Google. Identificaram-se 14 áreas de conhecimento, com predominância de autores que atuam nas áreas de Educação (118), Ciência da Computação (55) e Engenharias (29), enquanto as demais áreas contabilizam menos de 20\% dos autores (40). Cinco autores foram contabilizados em duas áreas (Ciência da Computação e Engenharia) e 4 autores não foram contabilizados por não ter sido possível localizar e/ou inferir a sua área de atuação. A Figura 1 ilustra essas constatações.

A partir de Schweitzer e Rodrigues (2013, p.157), pode-se inferir que este trabalho aborda, de fato, uma área de pesquisa emergente, cujas"características multidisciplinares apresentam desafios em seu mapeamento, pois envolvem pesquisadores com atuação em diferentes áreas, com formações distintas". Santos-Hermosa et al. (2012) confirmam que esta é uma área emergente, embora seja evidente a predominância da Educação, da computação e das Engenharias em relação às demais áreas. Por meio da caracterização de seus autores, verificou-se que eles provêm de áreas de conhecimento diversas, o que pode interferir nas consolidações teóricas sobre o tema.

\section{Análise de conteúdo dos artigos sobre OER}

Durante a etapa da organização da Análise de Conteúdo, obteve-se a versão digital completa dos artigos que compõem o corpus da pesquisa. Entretanto, 10, dos 115 artigos são publicações de periódicos cujo acesso é pago. Na ocasião da pesquisa, esses periódicos não constavam no Portal de Periódicos da Coordenação de Aperfeiçoamento de Pessoal de Nível Superior (Capes) e também não eram assinados pela Biblioteca Universi-

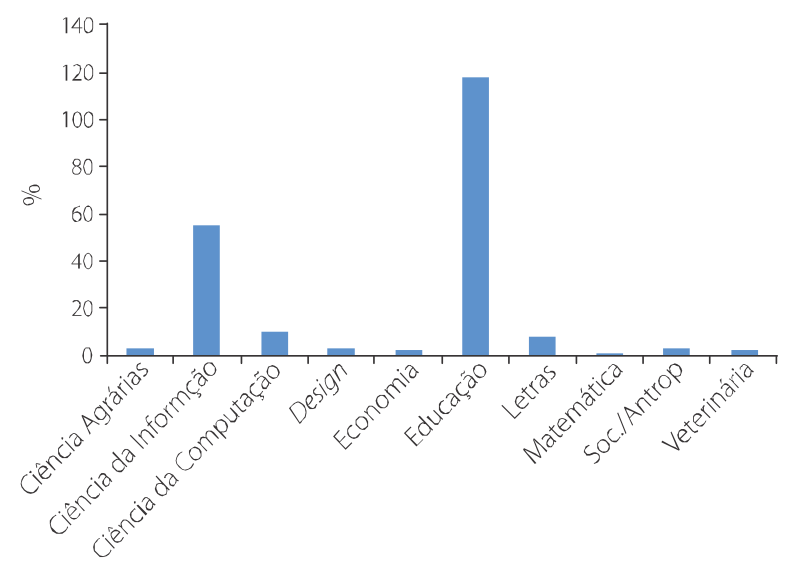

Figura 1. Área de conhecimento em que os autores atuam. Fonte: Dados da pesquisa. 
tária da Universidade Federal de Santa Catarina. A única exceção é o Teaching in Higher Education que, embora disponível no portal da Capes, tem período de embargo de 1 ano e 6 meses.

No levantamento efetuado, evidenciou-se que 99 artigos foram publicados em inglês, 8 em espanhol e 2 em português. É possível afirmar que o diálogo entre os pares nos canais formais se realiza por meio do idioma oficial da ciência, o inglês, que assegura a abrangência de público desejável à divulgação de resultados (VOLPATO, 2008). Meneghini (2013) reitera que o uso do inglês na comunicação científica abarca 97\% das publicações consideradas em seu estudo. Nesta pesquisa, 93\% dos artigos foram escritos em inglês.

As fichas documentais preenchidas com as informações oriundas dos artigos permitiram aferir que, dos 109 artigos verificados na análise de conteúdo: 3 (2\%) artigos não contêm resumo; 12 (11\%) artigos não contêm palavras-chave; e 70 (64\%) não têm uma seção nomeada "metodologia" ou"procedimentos metodológicos".

Constatou-se que os artigos reportam pesquisas, estudos e experiências de maneiras distintas. Por essa razão, dividiram-se os artigos conforme a sua abordagem - reflexões teóricas e relatos de experiências ou pesquisa: 26 (23\%) artigos foram classificados como reflexões teóricas, por abordarem o tema por meio do estudo da literatura e de sua crítica, exclusivamente; 83 (76\%) artigos foram classificados como aplicação ou relato de experiência, por descreverem iniciativas realizadas ou aplicações possíveis e/ou efetuadas.

Em seguida, as categorias foram criadas a partir das informações levantadas e codificadas após a análise integral dos artigos. Dessa forma, criaram-se as seguintes categorias:

a) Adaptação e reúso: processo por meio do qual um recurso pronto é editado ou adaptado para ser reutilizado em outro contexto educacional. Procedimento e/ou contexto em que um recurso pronto é reutilizado em outro contexto de educação;

b) Desafios: questões, dificuldades e críticas ao uso e/ou à fundamentação teórica dos OER;

c) Open Courseware: ferramenta por meio da qual as instituições disponibilizam seus materiais na Internet a fim de apoiar a educação aberta e a aprendizagem ao longo da vida;

d) Políticas de incentivo e sustentabilidade: políticas públicas institucionais que incentivam e fomentam o desenvolvimento e o uso de OER no âmbito da Educação. Modos como as iniciativas OER se mantêm e sustentam, especialmente dos aspectos financeiro, político e institucional;

e) Produção: modos por meio dos quais os OER são criados, desenvolvidos ou implementados;

f) Recuperação e repositórios: técnicas e abordagens para encontrar OER. Bases onde se armazenam os OER para sua posterior recuperação; e

g) Tecnologias: ferramentas e instrumentos em geral utilizados como OER propriamente ditos. Livros, áudios, apresentações.

A Tabela 2 detalha a quantidade de artigos classificados por categoria e abordagem, na ordem decrescente do número de artigos.

Tabela 2. Número de artigos por categoria e abordagem.

\begin{tabular}{|c|c|c|c|c|c|c|}
\hline \multicolumn{7}{|c|}{ Abordagem } \\
\hline Categoria & $\begin{array}{c}\text { Aplicações ou relatos } \\
\text { de experiências }\end{array}$ & $\%$ & Reflexões teóricas & $\%$ & Total & $\%$ \\
\hline Recuperação e repositórios & 20 & 18,0 & 3 & 2,0 & 23 & 22,0 \\
\hline Desafios & 9 & 8,0 & 12 & 11,0 & 21 & 19,0 \\
\hline Tecnologias & 17 & 15,0 & 1 & 0,9 & 18 & 16,0 \\
\hline Produção & 14 & 12,0 & 2 & 1,0 & 16 & 14,0 \\
\hline Políticas de incentivo e sustentabilidade & 8 & 7,0 & 7 & 6,0 & 15 & 13,0 \\
\hline Adaptação e reúso & 10 & 9,0 & 1 & 0,9 & 11 & 10,0 \\
\hline Open Courseware & 5 & 4,0 & 0 & 0,0 & 5 & 4,0 \\
\hline Total & 83 & 76,0 & 26 & 23,0 & 109 & 100,0 \\
\hline
\end{tabular}


A seguir, sintetizam-se as inferências conforme as categorias estabelecidas a partir dos trechos interpretados como mais significativos no corpus (Quadro 2).

\section{Conclusão}

Identificaram-se, por meio de levantamento, 115 artigos de 243 pesquisadores, publicados em 43 periódicos científicos nas bases da WoS. Os 43 periódicos científicos, cujos artigos publicados compõem o corpus da pesquisa, remontam a 16 países, a maioria (13 periódicos; 81\%) do Reino Unido.

Identificou-se a tendência de aumento da produção científica sobre Recursos Educacionais Abertos no
Ensino Superior, embora a do ano de 2014 não tenha sido acompanhada, possivelmente devido à data da coleta nas bases, em fevereiro de 2015. Os artigos identificados reiteram o crescimento do tema ao longo dos anos: inicia em 2008, com 3 artigos publicados, alcançando 33 publicações em 2013.

Constatou-se que a maior parte (29 periódicos; 67\%) dos periódicos que abordam o tema OER é de acesso pago ou por subscrição paga com opção de acesso aberto, concentrando $56 \%$ dos artigos em acesso restrito.

Verificou-se que o núcleo das publicações se concentra na área de Educação (26 periódicos e 95 artigos), Engenharia (4 periódicos e 10 artigos), Ciência e

Quadro 2. Categorias e inferências.

\begin{tabular}{|c|c|}
\hline Recuperação e repositórios & $\begin{array}{l}\text { É preciso que os OER possam ser facilmente recuperados e reutilizados em plataformas } \\
\text { distintas, razão pela qual a sua interoperabilidade é uma condição fundamental. A recuperação } \\
\text { de OER constitui uma tarefa desafiadora ante a heterogeneidade dos modelos adotados } \\
\text { pelos repositórios na atualidade. A variedade de padrões dificulta a interoperabilidade, cujo } \\
\text { objetivo é promover o acesso simultâneo aos dados contidos em diversos repositórios, de } \\
\text { forma a maximizar as buscas e reduzir o tempo de resposta. Além disso, consideram-se } \\
\text { melhores as buscas capazes de contemplar o nível de abertura, ou as formas de licença dos } \\
\text { recursos }\end{array}$ \\
\hline Desafios & $\begin{array}{l}\text { Os OER não são uma solução para os problemas educacionais. Conteúdo em si não é edu- } \\
\text { cação e, por isso, o papel do professor, nesse possível novo modelo pedagógico, é fundamental. } \\
\text { A questão do acesso à internet de banda larga é imprescindível para a utilização de OER. }\end{array}$ \\
\hline Tecnologias & $\begin{array}{l}\text { Os livros de licença aberta são uma opção significativamente econômica para estudantes e } \\
\text { instituições. O acesso livre aos OER não é condição suficiente, tampouco garantia de } \\
\text { aprendizagem. O potencial e a influência dos OER podem ser limitados pela tecnologia } \\
\text { utilizada na sua produção. }\end{array}$ \\
\hline Produção & $\begin{array}{l}\text { Os OER devem ser produzidos em contextos colaborativos, que lhes dará grande potencial de } \\
\text { especialização, e preconizar as licenças abertas, do tipo Creative Commons, o que permitirá a } \\
\text { continuidade do processo de produção, adaptação e possível aprimoramento. }\end{array}$ \\
\hline Políticas de incentivo e sustentabilidade & $\begin{array}{l}\text { Além de configurar mais possibilidade de acesso a conteúdos educacionais, a adoção de OER } \\
\text { pode ser uma decisão pela economia de recursos - por isso, interessa não apenas a insti- } \\
\text { tuições de educação a distância, mas também àquelas que ofertam educação presencial. } \\
\text { Para tanto, cabe adotar um modelo educacional cujas políticas institucionais incentivem e } \\
\text { reconheçam o uso dos OER por meio de políticas, diretrizes e financiamentos de incentivo à } \\
\text { produção qualificada e à sustentabilidade. }\end{array}$ \\
\hline Adaptação e reúso & $\begin{array}{l}\text { O idioma é a principal barreira ao reúso de OER; então, as adaptações se concentram majo- } \\
\text { ritariamente nas traduções. As questões culturais devem ser contempladas nas adaptações, } \\
\text { de forma que o reúso não configure mera exportação ou imperialismo cultural. }\end{array}$ \\
\hline Open Courseware & $\begin{array}{l}\text { São ferramentas importantes para aumentar a participação na educação superior formal, } \\
\text { apoiando, assim, a educação formal. As políticas de direitos autorais dos OCW, em sua } \\
\text { maioria, divergem daquela preconizada pelos OER, em geral, pois, embora permitam acesso } \\
\text { livre e gratuito aos recursos educacionais, isso não significa que os usuários fiquem livres de } \\
\text { responsabilidades e tampouco que possam reutilizá-los, adaptá-los e/ou remixá-los. }\end{array}$ \\
\hline
\end{tabular}

Notas: OER: Open Educational Resources; OCW: Open Courseware. 
Tecnologia (5 periódicos e 10 artigos), Ciência da Informação (5 periódicos e 7 artigos), Saúde (3 periódicos e 3 artigos), Humanas (3 periódicos e 3 artigos), Linguística (2 periódicos e 2 artigos) e Multidisciplinar (2 periódicos e 2 artigos), entre outras (9 periódicos e 11 artigos).

A concentração de títulos da área da Educação chega a $82 \%$ da amostra, com $28 \%$ deles publicados no periódico canadense International Review of Research in Open and Distance Learning. Com relação aos periódicos, identificou-se que 32\% são de acesso aberto, sendo $81 \%$ do Reino Unido e 60\% da área da Educação.

Identificaram-se 243 autores, ligados a 107 instituições de 36 países. Verificou-se que, da totalidade das instituições, 96 (89\%) são universitárias, revelando o protagonismo das universidades na produção de pesquisa, visto que apenas uma minoria dos autores não possui relação com a academia. Acerca da área de atuação dos autores, constatou-se que 48\% trabalham na área da Educação, 22\% na Ciência da Computação, 12\% nas Engenharias e $8 \%$ na Ciência da Informação. O Reino Unido concentra 22\% dos autores, seguido dos Estados Unidos (17\%) e Espanha (12\%).

Observou-se que as instituições que mais concentram pesquisadores sobre OER no Reino Unido, na Espanha e no Canadá, são todas especializadas em Educação a Distância: respectivamente, a Open University, a Universidad Nacional de Educación a Distancia e a Athabasca University. Esse fato demonstra a relevância dos OER nessa modalidade de ensino, tanto em razão de suas potencialidades, quanto pelo fato de esse tema decorrer de um anterior, que Ihe é afim, do âmbito da educação a distância: os objetos de aprendizagem.

\section{Referências}

AMIEL, T.; SOARES, T. C. O contexto da abertura: recursos educacionais abertos, cibercultura e suas tensões. Em Aberto, v. 28, n. 94, p. 109-122, 2015.

ABADAL, E. Acceso abierto a la ciencia. Barcelona: Editorial UOC, 2012. Disponible en: <http://diposit.ub.edu/dspace/ bitstream/2445/24542/1/262142.pdf>. Acceso en: 22 mai. 2013.

ABELSON, H. The Creation of OpenCourseWare at MIT. Journal of Science Education and Technology, v. 17, n. 2, p. 164-174, 2008.

ARAÚJO, C. A. Bibliometria: evolução história e questões atuais. Em Questão, v. 12, n. 1, p. 11-32, 2006.
Embora o periódico analisado que mais concentra artigos sobre o tema seja o canadense International Review of Research in Open and Distance Learning, da Athabasca University, esse país ocupa o quarto lugar na quantidade de autores que publicaram sobre OER nesta pesquisa: 10 autores (4\%), de 4 instituições, estando a maioria deles (6) concentrada na Athabasca University, especializada em educação a distância.

Com relação à recuperação de OER, inferiu-se que a interoperabilidade é uma condição fundamental ante a heterogeneidade dos modelos adotados pelos repositórios na atualidade. Serão melhores e mais úteis na busca de OER as publicações que contemplarem o nível e a abertura - ou sua forma de licença-dos recursos. Reconhece-se que apenas conteúdo não é educação - e, por isso, o papel do professor, nesse novo modelo baseado na autoria docente e nos OER, é fundamental e desafiador. Além disso, o acesso livre aos OER não é condição suficiente, tampouco garantia de aprendizagem.

Os Open Educational Resources podem configurar importantes meios para a viabilização da Educação Aberta, possibilitando inúmeras configurações de desenvolvimento de iniciativas no cenário da cultura digital. Seu foco é a abertura, e a partir dela decorre todo o seu potencial de democratização da educação e do conhecimento. Em contrapartida, os OER não são uma panaceia.

\section{Colaboradores}

Todos as autoras contribuíram na concepção e desenho do estudo, análise de dados e redação final.

BARDIN, L. Análise de conteúdo. São Paulo: Edições 70, 2011.

BRAGA, K. S. Aspectos relevantes para a seleção de metodologia adequada à pesquisa social em Ciência da Informação. In: MUELLER, S. M.P. (Org.). Métodos para a pesquisa em Ciência da Informação. Brasília: Thesaurus, 2007.

BUTCHER, N. A basic guide to Open Educational Resources. British: Commonwealth of Leaning, 2011. Available from: <http://www.col.org/resources/publications/Pages/detail. aspx?PID= 357>. Cited: Aug. 21, 2014.

CAMILLERI, A. F.; EHLERS, U. D; PAWLOWSKI, J. State of the art review of quality issues related to Open Educational Resources (OER). Luxembourg: Publications Office of the European Union, 
2014. Available from: <http://is.jrc.ec.europa.eu/pages/EAP/ documents/201405JRC88304.pdf>. Cited: Apr. 25, 2016.

CERVO, A. L.; BERVIAN, P. A.; SILVA, R. Metodologia científica. 6.ed. São Paulo: Pearson Prentice Hall, 2007.

CONONNAY, L. S.; POWELL, R. R. Basic research methods for librarians. Santa Barbara: Libraries Unlimited, 2010, p. 1-359.

COSTA, M. T. F. O uso de recursos educativos abertos (REA) como recursos didáticos: benefícios para alunos e professores: o caso do repositório científico de acesso aberto de Portugal. Liinc em Revista, v. 8, n. 2, p. 402-412, 2012.

CREATIVE COMMONS. About the licenses. MAITAIN VIEW: Creative Commons. Available from: $<$ http://creativecommons. org/licenses/?lang=pt_BR>. Cited: Mar. 23, 2015.

CRESWELL, J. W. Projeto de pesquisa: métodos qualitativo, quantitativo e misto. 3. ed. Porto Alegre: Artmed, 2010.

D'ANTONI, S. Introduction. In: D'ANTONI, S.; SAVAGE, C. (Org.). Open Educational Resources: Conversations in cyberspace. Paris: Unesco, 2009, p. 16-27.

DEIMANN, M.; FARROW, R. Rethinking OER and their use: Open education as bildung. International Review of Research in Open and Distance Learning, v. 14, n. 3, p. 344-360, 2013.

FERREIRA, A. G. C. Bibliometria na avaliação de periódicos científicos. DataGramaZero, v.11, n.3, 2010. Disponível em: <http://www.dgz.org.br/jun10/Art_05.htm>. Acesso em: 13 jun. 2012

GUÉDON, J. C. In Oldenburg's long shadow: Librarians, research scientists, publishers, and the control of scientific publishing. Washington, D.C.: Association of Research Libraries, 2001. Available from: <http://www.arl.org/storage/documents/ publications/in-oldenburgs-long-shadow.pdf > . Cited: Jan. 20, 2015.

GUTIÉRREZ, D. M.; IBARRA, A.; MONTOYA, M. S. Estrategias de comunicación para potenciar el uso de Recursos Educativos Abiertos (REA) a través de repositorios y metaconectores. Innovar, v. 24, n. 52, p. 67-78, 2014

LANE, A. The impact of openness on bridging educational digital divides. International Review of Research in Open and Distance Learning, v. 10, n. 5, p. 1-12, 2009.

MEADOWS, A. J. A comunicação científica. Brasília: Briquet de Lemos, 1999.

MENEGHINI, R. Seria hora de publicar mais em inglês? Química Nova, v. 36, n. 6, p. 755, 2013. Disponível em: <http://dx.doi.org/ 10.1590/S0100-40422013000600001>. Acesso em: 23 abr. 2016.

MUELLER, S. P. M. Literatura científica, comunicação científica e ciência da informação. In: TOUTAIN, L. M. B. (Org.). Para entender a Ciência da Informação. Salvador: EDUFBA, 2007. p. 125-144.
MULDER, J. Knowledge Dissemination in Sub-Saharan Africa: What Role for Open Educational Resources (OER)? (Master's Thesis International Relations) - International School for Humanities and Social Sciences). Amsterdam: 2008. University of Amsterdam. Available from: <http://www.gg.rhul.ac.uk/ ict4d/workingpapers/mulderOER.pdf>. Cited: Mar. 23, 2015.

PACKER, A. L. Os periódicos brasileiros e a comunicação da pesquisa nacional. Revista USP, n. 89, p. 26-61,2011. Disponível em: <http://rusp.scielo.br/scielo.php?script=sci_arttext\& pid $=$ S0103-99892011000200004\&lng $=$ pt\&nrm=iso $>$. Acesso em: 25 abr. 2016.

ROLFE, V. Open educational resources: Staff atitudes and awarenses. Resources in Learning Technology, v. 20, 2012. Available from: <http://www.researchinlearningtechnology. net/index.php/rlt/article/view/14395 > . Cited: Sept. 20, 2015. doi: 10.3402/rlt.v20i0/14395

ROSSINI, C.; GONZALEZ, C. REA: o debate em política pública e as oportunidades para o mercado. In: SANTANA, B.; ROSSINI, C.; PRETTO, N. L. (Org.). Recursos Educacionais Abertos: práticas colaborativas políticas públicas. Salvador: EDUFBA, 2012.

SANTOS, A. I. Recursos Educacionais Abertos no Brasil: o estado da arte, desafios e perspectivas para o desenvolvimento e inovação. São Paulo: Comitê Gestor da Internet no Brasil, 2013.

SANTOS-HERMOSA, G.; FERRAN-FERRER, N.; ABADAL, E. Recursos educativos abiertos: repositorios y uso. El Profesional de la Información, v. 21, n. 2, p. 136-145, 2012.

SCHWEITZER, F.; RODRIGUES, R. S. Produção científica em áreas multidisciplinares: educação a distância no Brasil. Revista da Faculdade de Biblioteconomia e Comunicação da UFRGS, v. 19, n. 1, p. 156-172, 2013.

TOVAR, E.; PIEDRA, N. Guest editorial: Open educational resources in engineering education: Various perspectives opening the education of engineers. IEEE Transactions on Education, v. 57, n. 4, p. 213-219, 2014.

UNITED NATIONS EDUCATIONAL, SCIENTIFIC AND CULTURAL ORGANIZATION. Educational: Forum on the impact of open courseware for Higher Education in developing countries. Paris: Unesco, 2002. Available from: <http://unesdoc. unesco.org/images/0012/001285/128515e.pdf>. Cited: Mar. 26, 2016.

VANTI, N. Indicadores web e sua aplicação à produção científica disponibilizada em revistas eletrônicas. In: Ferreira, S.M.S.P.; Targino, M.G. (Org.). Acessibilidade e visibilidade de revistas científicas eletrônicas. São Paulo: Senac, 2010.

VOLPATO, G. Publicação científica. São Paulo: Cultura Acadêmica, 2008.

WHITLEY, R. The intellectual and social organization of the sciences. $2^{\text {nd }}$ ed. New York: Oxford University Press, 2000. 
